Products and Services In Practice is provided to readers using text and images from the manufacturer, supplier or distributor and does not imply endorsement by $B D J$ In Practice. Normal and prudent research should be exercised before purchase or use of any product mentioned.

Please send product and services news through to David Westgarth, BDJ In Practice via: David.Westgarth@bda.org

\section{Sterile needn't}

\section{mean boring}

The new Lisa type B vacuum steriliser from $\mathrm{W} \& \mathrm{H}$ is a powerful, ergonomic, professional-level benchtop steam steriliser with a full suite of helpful features.

In addition to its easy-to-use colour touchscreen and full, integrated traceability handling, Lisa works with the new Lisa Mobile App - enabling you to monitor the machine in real-time from your smartphone. Patented Eco Dry+ technology modifies the drying time to correspond to the load for maximum efficacy - saving you time and energy.

With outstanding, high performance, exceptional connectivity and a blistering turnaround of just 30 minutes for an average load, Lisa is the steriliser you've been looking for.

To find out more visit www.wh.com/ en_uk, call 01727874990 or email office. uk@wh.com.

\title{
For a powerful and personal patient experience
}

Align Technology, Inc. (NASDAQ: ALGN) has announced the launch of its latest digital tool for general dentists and especially Invisalign Go Providers. The ClinCheck In-face Visualisation tool is an enhanced digital tool, which allows for an image of the patient's face to be incorporated into their treatment plan.

Actively engaging patients at every stage of their treatment is now simpler than ever before. By helping patients visualise their future smile, general dentists can clearly communicate the benefits of teeth straightening and guide patients towards the treatment choice most suitable for them.

Align Technology's latest enhancement to the 3D Clincheck software will take patient smile visualisation to the next level by helping dentists generate a powerful, clinical visualisation of the patient's teeth in their face. It will also enable general dentists to showcase benefits of teeth straightening and guide their patients towards the most suitable treatment choices. This is made possible with the use of the Invisalign Photo Uploader application (available on iPhone and Android) to capture digital photos of the patient. The iTero intraoral scanner can then capture digital records in a more convenient and efficient way.

Align's proprietary ClinCheck software has long provided practitioners with $3 \mathrm{D}$ imaging of tooth movements from start to finish so they have options for the patient treatment journey. Now, with the added benefit of the 'In-face' Visualisation, ClinCheck allows dentists to customise treatment plans according to the patient's facial feature to make the visualisation more natural.

The ClinCheck In-face Visualisation tool is now available for general practitioners using the Invisalign Go system in the United Kingdom and Ireland.

For more information, visit www. invisalign-go.co.uk.

\section{A polished finish}

Zirconia restorations look their best once they have been polished to achieve a naturallooking lustre. However, due to the unique qualities of the material they require different tools in order to achieve a lasting shine.

That's why, when clinicians choose the new Monolith Full-Contour Zirconia

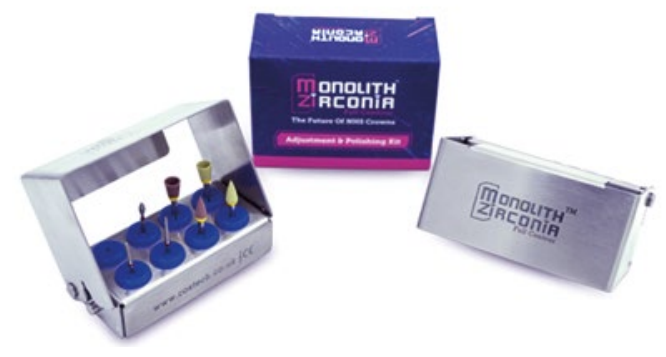

from CosTech Dental Laboratory, the lab offers a specifically designed Polishing Kit. This kit contains everything that professionals need to achieve a beautiful, lasting gloss on these restorations as well to perform minor adjustments on the material to prevent it being abrasive against opposing teeth.

Monolith restorations are priced at just $£ 29.95$ per unit, including free delivery to and from the lab. The Monolith Adjustment \& Polishing Kits are priced at $£ 149.95$.

To find out more, contact CosTech by visiting www.costech.co.uk or call 01474320076 . 